\title{
Taenia saginata
}

National Cancer Institute

\section{Source}

National Cancer Institute. Taenia saginata. NCI Thesaurus. Code C125960.

A species of parasitic cestodes in the genus Taenia that is acquired through the ingestion of infected bovine tissue. Humans are the definitive host of the adult cestodes, resulting in taeniasis. 